Journal of Agricultural Sciences
(Tarim Bilimleri Dergisi)

\title{
Aphid Species, Their Natural Enemies in Vegetables from Erzincan, Turkey: First Record of the Parasitoid Wasp Aphelinus mali (Haldeman) parasitizing Lipaphis erysimi (Kaltenbach)*
}

\author{
İsmail ALASERHAT ${ }^{a} \mathbb{D}$, Adnan CANBAY ${ }^{b} \mathbb{D}$, Ișıl ÖZDEMİR ${ }^{c}$ \\ ${ }^{a}$ Directorate of Horticultural Research Institute, 24060, Erzincan, TURKEY \\ ${ }^{b}$ Directorate of Apricot Research Institute, 44090, Malatya, TURKEY \\ ${ }^{c}$ Directorate of Plant Protection Central Research Institute, 06172, Ankara, TURKEY \\ ARTICLE INFO \\ Research Article \\ Corresponding Author: İsmail ALASERHAT, E-mail: i_alaserhat36@hotmail.com \\ Received: 10 June 2019 / Revised: 03 September 2019 / Accepted: 09 September 2019 / Online: 18 January 2021
}

\section{ABSTRACT}

Aphid species, their parasitoids and predators on vegetables (bean, cucumber, eggplant, melon, okra, pepper, pumpkin, tomato and watermelon) grown in Erzincan province, Turkey were determined. Surveys were carried out at weekly intervals from the seedling period until the end of the harvest period in Central and Üzümlü districts of Erzincan province in 2014-2016. 30-40 plants were randomly selected in the surveys and all parts of plants were investigated with a magnifier. Ten aphid, seventeen predator and seven parasitoids species were found According to results, Lipaphis erysimi (Kaltenbach) (Hemiptera:
Aphididae) was first recorded as a host of Aphelinus mali (Haldeman) (Aphelinidae: Aphelininae) in the world. In addition, parasitism rates of aphids were also established. The mean parasitism rate changed between $17.5 \%$ in 2015 and $4.39 \%$ in 2016 . The relationship between parasitism rate, total parasitized aphid number and total aphid number were found. According to the analysis result, there was a very weak or a high positive correlation $(r=0.126-0.721)$ between total aphid number and total parasitized (mummified) aphid number, and not a correlation existed between parasitism rate and total aphid number as for years.

Keywords: Aphid, Natural enemy, Parasitism rate, Vegetable, Turkey

(c) Ankara University, Faculty of Agriculture

\section{Introduction}

One of the food groups that people need for healthy and balanced nutrition is vegetables and fruits. Vegetables; vitamins, minerals and other ingredients in terms of human nutrition are a very important place (Thompson \& Kelly 1990).

Vegetable production could be decreased by few pest organisms. One of the most important of these pests is aphids. Aphids (Hemiptera: Aphididae) are recognized worldwide as economically important (Remaudière \& Autrique 1985). Aphid species are polyphagous, feed on plant sap and multiply rapidly under favorable conditions. They feed on the leaves, shoots, branches, stems, fruits and roots of plants and cause serious damage and also lead to the formation of fumagine. This formation reduces the photosynthesis and respiration capacity of plants (Lodos 1986; Ölmez Bayhan \& Ulusoy 2002; Tepecik et al. 2011). In addition to these damages, aphids also vectors to viruses and other similar organisms, which are often more important than other damages (Lodos 1986; Matheus 1993).

It is reported that there are 510 genus (that have reached 5.000 species) of aphids on the earth (Blackman \& Eastop 2018). Important vegetable pest species of the Aphididae family (Hemiptera) are mainly belong to the genera Aphis (Linnaeus 1758), Aulacorthum (Mordvilko 1914), Brevicoryne (van der Goot 1915), Macrosiphum (Passerini 1860) and Myzus (Passerini 1860). Among these species, Aulacorthum solani (Kaltenbach 1843), Aphis fabae (Scopoli 1763), Aphis gossypii (Glover 1877), Brevicoryne brassicae (Linnaeus 1758), Macrosiphum euphorbiae (Thomas 1878) and Myzus persicae (Sulzer 1776) are the most important vegetable pest species in Turkey (Düzgüneş \& Tuatay 1956; Çanakçığlu 1975; Göksu \& Atak 1976; Ölmez Bayhan 2000; Toros et al. 2002; Sangün 2010). 
Although many studies have been carried out on vegetable aphids in Turkey, no comprehensive study has been done on vegetable aphids in Erzincan until now. This study was carried out to establish aphid species and their parasitoids, predators, parasitism rates on vegetables growing in Erzincan province. Thus, within the scope of integrated pest management and organic vegetable cultivation, basic data of biological control against aphids were found out. Potential natural enemies determined as a result of this work, will be tackle of later, and it will lead to subjects such as increasing of natural enemy activity and mass production.

\section{Material and Methods}

Aphid species and their parasitoids, predators were collected on plants of Abelmoschus esculentus L., Capsicum annuum L., Citrullus lanatus Thunb., Cucurbita pepo L., Cucumis sativus L., Cucumis melo L., Lycopersicon esculentum Miller, Phaseolus vulgaris L., Solanum melongena L. grown in greenhouse or open field in Erzincan province.

To determine aphid species on vegetable plants in Center and Üzümlü district of Erzincan province, surveys were carried out at weekly intervals from the seedling period until the end of the harvest period in 2014 and 2016. In surveys, according to the field size 30-40 plants selected by chance and all part of the plants were examined by means of magnifier. Samples collected to determine aphid species were brought to laboratory for diagnosis by puting into codded tubes including $70 \%$ ethyl alcohol.

In order to determine the parasitoids and predators, firstly vegetables containing aphids had been observed and then detected parasitized aphids and predators were collected and they were brought to the laboratuvary for further identification with their host aphids. While collecting immature stages of parasitized, suspect aphids thought to be parasitized and both predators and their hosts from vegetables, they carefully were swathed with paper towels and placed into polyethylene bags. Immediateley, samples were placed in a portable refrigerator to prevent damage from the hot, and then brought to the laboratory in order to rear adults.

Non-adult (immature stages) predators collected were reared to become adults in a culture box (the dimensions of the culture box was 30x35x $15 \mathrm{~cm}$ and the top of them surrounded by tulle) in laboratory and they were daily controled. When it was needed, aphid infected fresh plant shoots were added into the culture box and then, obtained adult predators were diagnosed (Yumruktepe 1993; Aslan 2002). After cleaning all the pests except from the aphids, suspect aphids and aphid mummies were placed in parasitoid extraction boxes. Following that daily emerging parasitoids were transferred into eppendorf tube with $95 \%$ ethyl alcohol and diagnosed (Yumruktepe 1993).

All vegetable plants were randomly sampled to establish the rate of natural parasitism. During this study, while determining the rate of parasitism, total parasitized individual numbers obtained from the colonies including mummy was compared with total aphid numbers in the sample (Praslicka \& Huszar 2005).

In this study, correlation and regression analyses were done on data by using SPSS 17.0 packet program to determine whether there was a relationship between total parasitized (mummified) aphids and total aphids and between parasitism rate and total aphids.

\section{Results and Discussion}

In conclusion, it was found that there were ten aphid species, Acyrthosiphon pisum (Harris), Aphis craccivora Koch, Aphis fabae Scopoli, Aphis gossypii Glover, Aphis nasturtii Kaltenbach, Aphis spiraecola Patch, Aulacorthum solani Kaltenbach, Lipaphis erysimi (Kaltenbach), Myzus (Nectarosiphon) persicae Sulzer and Macrosiphum euphorbiae (Thomas) (Aphididae) on grown vegetables in Center and Üzümlü district of Erzincan province in 2014-2016 (Table 1). It was found that aphids on Capsicum annuит L., Cucurbita pepo L., Phaseolus vulgaris L. was more intense in terms of distribution and intensity among other vegetable plants. The species with the highest number of host plants were identified as A. gossypii and M. persicae. Acyrthosiphon pisum, Aphis nasturtii and L. erysimi respectively determined only on the P. vulgaris, C. pepo and Cucumis sativus L. Holman (2009), found that total 42 aphids species on vegetables in Palearctic region and reported that these vegetable plants (bean, cucumber, eggplant, melon, okra, pepper, pumpkin, tomato and watermelon) were the host of Acyrthosiphon pisum, Aphis craccivora, A. fabae, A. gossypii, A. nasturtii, A. spiraecola, Aulacorthum solani, L. erysimi, M. persicae and M. euphorbiae. Bayram \& Bayhan (2016), determined that A. gossypii species were found commonly and consisting of high population on watermelons in Diyarbakır province in Turkey. Alaserhat \& Canbay (2017), found that A. craccivora, A. gossypii, A. fabae and M. persicae on pepper plant (C. апnиum) in Erzincan province in Turkey. 
Table 1- The aphid species detected in vegetables in Erzincan province in 2014-2016

\begin{tabular}{|c|c|}
\hline Host plants & Aphid species \\
\hline \multirow{4}{*}{ Phaseolus vulgaris L. } & Acyrthosiphon pisum (Harris 1776) \\
\hline & Aphis craccivora Koch, 1854 \\
\hline & Aphis fabae Scopoli, 1763 \\
\hline & Myzus (Nectarosiphon) persicae (Sulzer 1776) \\
\hline \multirow{3}{*}{ Cucumis sativus L. } & Aphis gossypii Glover, 1877 \\
\hline & Aphis spiraecola Patch, 1914 \\
\hline & Lipaphis erysimi (Kaltenbach 1843) \\
\hline \multirow{2}{*}{ Solanum melongena L. } & Aphis gossypii Glover, 1877 \\
\hline & Myzus (Nectarosiphon) persicae (Sulzer 1776) \\
\hline \multirow{2}{*}{ Cucumis melo L. } & Aphis gossypii Glover, 1877 \\
\hline & Aulacorthum solani (Kaltenbach 1843) \\
\hline \multirow{2}{*}{ Abelmoschus esculentus L. } & Aphis craccivora Koch, 1854 \\
\hline & Aphis gossypii Glover, 1877 \\
\hline \multirow{4}{*}{ Capsicum annuum $\mathrm{L}}$. & Aphis craccivora Koch, 1854 \\
\hline & Aphis fabae Scopoli, 1763 \\
\hline & Macrosiphum euphorbiae (Thomas 1878) \\
\hline & Myzus (Nectarosiphon) persicae (Sulzer 1776) \\
\hline \multirow{4}{*}{ Cucurbita pepo L. } & Aphis gossypii Glover, 1877 \\
\hline & Aphis nasturtii Kaltenbach, 1843 \\
\hline & Aulacorthum solani (Kaltenbach 1843) \\
\hline & Myzus (Nectarosiphon) persicae (Sulzer 1776) \\
\hline \multirow{3}{*}{ Lycopersicon esculentum Miller } & Aphis spiraecola Patch, 1914 \\
\hline & Macrosiphum euphorbiae (Thomas 1878) \\
\hline & Myzus (Nectarosiphon) persicae (Sulzer 1776) \\
\hline \multirow{3}{*}{ Citrullus lanatus (Thunb.) } & Aphis fabae Scopoli, 1763 \\
\hline & Aphis gossypii Glover, 1877 \\
\hline & Myzus (Nectarosiphon) persicae (Sulzer 1776) \\
\hline
\end{tabular}

As a result of this study, seventeen predator species viz. Chrysoperla carnea (Stephens) (Neuroptera: Chrysopidae), Deraeocoris (Camptobrochis) punctulatus (Fallen), Deraeocoris (Camptobrochis) serenus (Douglas \& Scott) (Hemiptera: Miridae), Orius (Orius) niger (Wolff) (Hemiptera: Anthocoridae), Adalia bipunctata (L.), Adalia decempunctata (L.), Adalia fasciatopunctata revelierei Mulsant, Coccinella septempunctata (L.), Hippodamia (Adania) variegata (Goeze), Exochomus nigromaculatus (L.), Oenopia (Synharmonia) conglobata (L.), Stethorus punctillum Weise, Psyllobora vigintiduopunctata (L.) (Coleoptera: Coccinellidae), Episyrphus balteatus De Geer, Sphaerophoria scripta (L.), Scaeva dignota (Rondani) (Diptera: Syrphidae) and Leucopis sp. (Diptera: Chamaemyiidae) were identified on aphid species found on the study (Table 2). While determining these species, it was observed that adult and pre-adult stages of predator species were fed directly on aphid species found on the study. In previous studies were done on these aphid species in Turkey, many other predatory species were determined including predator species were found in our study (Düzgüneş et al. 1982a,b; Zeren \& Düzgüneş 1983; Ölmez Bayhan \& Ulusoy 2002; Aslan \& Uygun 2005; Ayyıldız \& Atlıhan 2006; Aslan \& Uygun 2007; Güleç 2011; Alaserhat 2015; Alaserhat \& Canbay 2017). In addition, it was reported on the world that Chrysoperla carnea, Chrysoperla pallens (Rambur) (Neuroptera: Chrysopidae), Geocoris pulvisculatus Distant (Hemiptera: Lygaeidae), A. bipunctata, A. fasciatopunctata revelierei, Brumoides sturalis (F.), Coccinella septempunctata, Cheilomenes sexmaculata (F.), Coccinella undecimpunctata L., Coccinella transversalis F., Harmonia axyridis Pallas, Hippodamia dimidiata (F.), Hippodamia (Adania) variegata, Menochilus sexmaculatus (F.), Microaspis discolor (F.), Micromus variegatus (F.), Oenopia kirbyi Muls., Oenopia quadripunctata Kapur, Oenopia sauzeti Muls., Pania luteopustulata (Muls.), Propylea quatuordecimpunctata (L.), Pseudoaspidimerus circumflexus (Motschulsky), Scymnus (Pullus) castaneus (Sicard), Scymnus guimeti Muls., Scymnus pyrocheilus Muls., Scymnus quadrillum (Motschulsky), Scymnus xerampelinus Muls., Spitocaria bisellata (Muls.) (Coleoptera: Coccinellidae), E. balteatus, Scaeva pyrastri L., (Diptera: Syrphidae) fed on these aphid species found on the study (Agarwala \& Ghosh 1988; Singh \& Bali 1993; Irshad 2001; Brown \& Mathews 2008; Guo et al. 2008; Sapathi 2009; Chaudhary \& Singh 2012; Jaferi 2013; Vandereycken et al. 2015; Rocca \& Messelink 2017). 
Table 2- Predators of aphid species found on vegetables in Erzincan province in 2014-2016

\begin{tabular}{|c|c|c|}
\hline \multirow{2}{*}{ Aphid species } & \multicolumn{2}{|r|}{ Predator species } \\
\hline & Familia & Species \\
\hline \multirow{5}{*}{ Acyrthosiphon pisum (Harris 1776) } & Anthocoridae & Orius (Orius) niger (Wolff) \\
\hline & Miridae & Deraeocoris (Camptobrochis) punctulatus (Fallen) \\
\hline & & Coccinella septempunctata (Linnaeus) \\
\hline & Coccinellidae & Hippodamia variegata (Goeze) \\
\hline & & Oenopia (Synharmonia) conglabata (Linnaeus) \\
\hline \multirow{8}{*}{ Aulacorthum solani (Kaltenbach 1843) } & Anthocoridae & Orius (Orius) niger (Wolff) \\
\hline & Miridae & Deraeocoris (Camptobrochis) punctulatus (Fallen) \\
\hline & & Adalia bipunctata (Linnaeus) \\
\hline & & Adalia fasciatopunctata revelierei Muls. \\
\hline & Coccinellidae & Coccinella septempunctata (Linnaeus) \\
\hline & & Hippodamia variegata (Goeze) \\
\hline & Syrphidae & Scaeva dignota (Rondani) \\
\hline & Chamaemyiidae & Leucopis sp. \\
\hline \multirow{5}{*}{ Aphis craccivora Koch, 1854} & Anthocoridae & Orius $($ Orius $)$ niger $($ Wolff) \\
\hline & Miridae & Deraeocoris (Camptobrochis) punctulatus (Fallen) \\
\hline & & Coccinella septempunctata (Linnaeus) \\
\hline & Coccinellidae & Hippodamia variegata (Goeze) \\
\hline & & Oenopia (Synharmonia) conglabata (Linnaeus) \\
\hline \multirow{4}{*}{ Aphis fabae Scopoli, 1763} & Anthocoridae & Orius (Orius) niger (Wolff) \\
\hline & Miridae & Deraeocoris (Camptobrochis) punctulatus (Fallen) \\
\hline & Chrysopidae & Chrysoperla carnea (Stephens) \\
\hline & Coccinellidae & $\begin{array}{l}\text { Coccinella septempunctata (Linnaeus) } \\
\text { Hippodamia variegata (Goeze) }\end{array}$ \\
\hline \multirow{15}{*}{ Aphis gossypii Glover, 1877} & Anthocoridae & Orius (Orius) niger (Wolff) \\
\hline & Miridae & Deraeocoris (Camptobrochis) punctulatus (Fallen) \\
\hline & & Adalia bipunctata (Linnaeus) \\
\hline & & Adalia decempunctata (Linnaeus) \\
\hline & & Adalia fasciatopunctata revelierei Muls. \\
\hline & & Coccinella septempunctata (Linnaeus) \\
\hline & Coccinellidae & Exochomus nigromaculatus (Goeze) \\
\hline & & Hippodamia variegata (Goeze) \\
\hline & & Oenopia (Synharmonia) conglabata (Linnaeus) \\
\hline & & Psyllobora vigintiduopunctata (Linnaeus) \\
\hline & & Stethorus punctillum Weise \\
\hline & & Episyrphus balteatus De Geer \\
\hline & Syrphidae & Scaeva dignota (Rondani) \\
\hline & & Sphaerophoria scripta (Linnaeus) \\
\hline & Chamaemyiidae & Leucopis sp. \\
\hline \multirow{7}{*}{ Aphis nasturtii Kaltenbach, 1843} & Miridae & Deraeocoris (Camptobrochis) punctulatus (Fallen) \\
\hline & & Adalia bipunctata (Linnaeus) \\
\hline & & Adalia decempunctata (Linnaeus) \\
\hline & Coccinellidae & Coccinella septempunctata (Linnaeus) \\
\hline & & Hippodamia variegata (Goeze) \\
\hline & Syrphidae & Scaeva dignota (Rondani) \\
\hline & Chamaemyiidae & Leucopis sp. \\
\hline Aphis spiraecola Patch, 1914 & Miridae & Deraeocoris (Camptobrochis) serenus (Douglas \& Scott) \\
\hline Lipaphis erysimi (Kaltenbach 1843) & Coccinellidae & $\begin{array}{l}\text { Coccinella septempunctata (Linnaeus) } \\
\text { Hippodamia variegata (Goeze) }\end{array}$ \\
\hline \multirow{3}{*}{ Macrosiphum euphorbiae (Thomas 1878) } & Miridae & Deraeocoris (Camptobrochis) serenus (Douglas \& Scott) \\
\hline & Coccinellidae & Coccinella septempunctata (Linnaeus) \\
\hline & & Hippodamia variegata (Goeze) \\
\hline \multirow{13}{*}{ Myzus (Nectarosiphon) persicae (Sulzer 1776) } & Anthocoridae & Orius (Orius) niger (Wolff) \\
\hline & Miridae & Deraeocoris (Camptobrochis) serenus (Douglas \& Scott) \\
\hline & Mliridae & Deraeocoris (Camptobrochis) punctulatus (Fallen) \\
\hline & Chrysopidae & Chrysoperla carnea (Stephens) \\
\hline & & Adalia bipunctata (Linnaeus) \\
\hline & & Adalia decempunctata (Linnaeus) \\
\hline & & Coccinella septempunctata (Linnaeus) \\
\hline & Coccinellidae & Hippodamia variegata (Goeze) \\
\hline & & Oenopia (Synharmonia) conglabata (Linnaeus) \\
\hline & & Stethorus punctillum Weise \\
\hline & & Episyrphus balteatus De Geer \\
\hline & Syrphidae & Scaeva dignota (Rondani) \\
\hline & Chamaemyiidae & Leucopis sp. \\
\hline
\end{tabular}


Aphelinus mali (Haldeman) (Aphelinidae: Aphelininae), Aphidius ervi Haliday, Aphidius colemani Viereck, Aphidius matricariae Haliday, Lysiphlebus fabarum (Marshall), Binodoxys angelicae (Haliday) and Praon volucre (Haliday) (Braconidae: Aphidiinae) were found on aphids as parasitoids (Table 3). According to earlier studies conducted in Turkey it was determined that Binodoxys acalephae (Marshall), Praon dorsale (Haliday) and P. volucre (Braconidae: Aphidiinae) were parazitoids of A. pisum; Aphelinus sp. (Aphelinidae: Aphelininae), A. colemani, B. acalephae, B. angelicae, Ephedrus persicae Froggatt, Lysiphlebus cardui (Marshall) and L. fabarum (Braconidae: Aphidiinae) were parazitoids of A. craccivora; Aphelinus sp. (Aphelinidae: Aphelininae), Adialytus ambiguus (Haliday), A. colemani, A. matricariae, B. acalephae, B. angelicae, Diaeretiella rapae (M'Intosh), L. fabarum and P. volucre (Braconidae: Aphidiinae) were parazitoids of A. fabae; Aphelinus sp. (Aphelinidae: Aphelininae), A. colemani, A. matricariae, B. angelicae, Lipolexis gracilis (Foerster), L. fabarum and $P$. volucre (Braconidae: Aphidiinae) were parazitoids of A. gossypii; Aphelinus sp. (Aphelinidae: Aphelininae), A. colemani, P. volucre, B. angelicae, E. persicae, Lysiphlebus ambiguus Halliday and L. fabarum (Braconidae: Aphidiinae) were parazitoids of A. spiraecola; Aphelinus asychis Walker, (Aphelinidae: Aphelininae), Adialytus ambiguus, Aphidius abjectus (Haliday), Aphidius funebris Mackauer, A. colemani, A. ervi, A. matricariae, B. angelicae, Ephedrus cerasicola Stary, E. persicae, P. volucre, Lipolexis gracilis, D. rapae, Lysiphlebus cardui (Marshall) and L. fabarum (Braconidae: Aphidiinae) were parazitoids of M. persicae; Adialytus salicaphis (Fitch), P. volucre and B. angelicae (Braconidae: Aphidiinae) were parazitoids of M. euphorbiae (Düzgüneş et al. 1982b; Zeren \& Düzgüneş 1983; Ayyıldız \& Atlıhan 2006; Güleç 2011; Alaserhat 2015; Alaserhat \& Canbay 2017). Furthermore, it was reported that numerous parasitoid species (including these species) were determined on aphids in the world (Atwal et al. 1971; Singh 1980; Tomanovic et al. 1998; Stary et al. 2000; Wiackowski et al. 2001; Kavallieratos et al. 2004; Rakhshani et al. 2005, Tomanovic et al. 2005; Kavallieratos et al. 2006; Stary 2006; Rakhshani et al. 2007; Talebi et al. 2009; Kavallieratos et al. 2010; Mossadegh et al. 2011; Dassonville et al. 2012; Nazari et al. 2012; Barahoei et al. 2013; Rakhshani et al. 2013; Taheri \& Rakhshani 2013; Anonymous 2018).

Table 3- Aphid and their parasitoid species on vegetables in Erzincan province in 2014-2016

\begin{tabular}{|c|c|c|}
\hline \multirow{2}{*}{ Aphid species } & \multicolumn{2}{|c|}{ Parasitoid species } \\
\hline & Subfamily & Species \\
\hline Aulacorthum solani (Kaltenbach) & Aphidiinae & Aphidius colemani Viereck \\
\hline Aphis craccivora Koch & Aphidiinae & Aphidius ervi Haliday \\
\hline Aphis fabae Scopoli & Aphidiinae & $\begin{array}{l}\text { Aphidius matricariae Haliday } \\
\text { Lysiphlebus fabarum (Marshall) } \\
\text { Praon volucre (Haliday) }\end{array}$ \\
\hline Aphis gossypii Glover & Aphidiinae & $\begin{array}{l}\text { Aphidius colemani Viereck } \\
\text { Aphidius matricariae Haliday } \\
\text { Binodoxys angelicae (Haliday) } \\
\text { Praon volucre (Haliday) }\end{array}$ \\
\hline Aphis spiraecola Patch & Aphidiinae & Praon volucre (Haliday) \\
\hline Lipaphis erysimi (Kaltenbach) & $\begin{array}{l}\text { Aphidiinae } \\
\text { Aphelininae }\end{array}$ & $\begin{array}{l}\text { Aphidius matricariae Haliday } \\
\text { Aphelinus mali (Haldeman) }\end{array}$ \\
\hline Macrosiphum euphorbiae (Thomas) & Aphidiinae & Praon volucre (Haliday) \\
\hline Myzus (Nectarosiphon) persicae (Sulzer) & Aphidiinae & $\begin{array}{l}\text { Aphidius colemani Viereck } \\
\text { Aphidius ervi Haliday } \\
\text { Aphidius matricariae Haliday } \\
\text { Praon volucre (Haliday) } \\
\text { Lysiphlebus fabarum (Marshall) }\end{array}$ \\
\hline
\end{tabular}

Two parasitoid species viz. Aphelinus mali (Aphelinidae: Aphelininae) and Aphidius matricariae (Braconidae: Aphidiinae) were determined on L. erysimi. Aphidius sp., A. colemani, Aphidius hortensis Marshall, A. matricariae, Aphidius rosea Haliday, Binodoxys brevicornis (Haliday), Binodoxys indicus (Subba Rao \& Sharma), Diaeretiella rapae, Ephedrus sp., Ephedrus laevicollis (Thomson), Ephedrus plagiator (Nees), Lipolexis gracilis, Lysiphlebus fabarum, Lysiphlebus testaceipes (Cresson) and Praon volucre (Braconidae: Aphidiidae) are pointed out as parasitoids of L. erysimi (Atwal et al. 1971; Singh 1980; Stary \& Ghosh 1983; Stary et al. 2000; Stary 2006; Talebi et al. 2009; Mossadegh et al. 2011; Rakhshani et al. 2013). Consequence, Aphelinus mali (Aphelinidae: Aphelininae) was first recorded as a parasitoid's of Lipaphis erysimi (Hemiptera: Aphididae) in the world.

Aphids' parasitism rates in 2015 are given (Table 4). Starting from the first week of July and continued until the first week of November, natural parasitism with aphids on vegetables was observed. At the end of the vegetation, the maximum parasitism was recorded (third week of October-first week of November). The highest parasitism rate was recorded as $84.93 \%$ on 21 October 2015 with cultured sample, while the mean rate of parasitism was $17.15 \%$.

After analyzing the 2015 data, it was found that, there was a very weak positive correlation $(r=0.126)$ between the the number of total parasitized (mummified) aphids and number of total aphids. Also, there was not a correlation between the parasitism rate and the number of total aphids. Regression analysis was performed on the data to detect the relationship between the number of total parasitized (mummified) aphids and the number of total aphids and a linear relationship between them was found (Figure 1). 
Table 4- The rate of parasitism of aphids on vegetables in 2015 in Erzincan province

\begin{tabular}{|c|c|c|c|c|c|}
\hline Host plant & $\begin{array}{l}\text { Date of } \\
\text { sampling }\end{array}$ & Aphid species & $\begin{array}{c}\text { Total } \\
\text { aphid } \\
\text { number }\end{array}$ & $\begin{array}{c}\text { Total } \\
\text { parasitized } \\
\text { (mummified) } \\
\text { aphid number }\end{array}$ & $\begin{array}{l}\text { Parasitism } \\
\text { rates }(\%)\end{array}$ \\
\hline \multirow{2}{*}{ Abelmoschus esculentus } & 15.07 .2015 & Aphis gossypii & 350 & 18 & 5.14 \\
\hline & 28.07.2015 & Aphis gossypii & 620 & 38 & 6.13 \\
\hline Capsicum annuит & 03.11 .2015 & $\begin{array}{l}\text { Aphis fabae } \\
\text { Myzus (Nectarosiphon) persicae }\end{array}$ & 150 & 80 & 53.33 \\
\hline Lycopersicon esculentum & 21.10 .2015 & $\begin{array}{l}\text { Aphis spiraecola } \\
\text { Macrosiphum euphorbiae }\end{array}$ & 73 & 62 & 84.93 \\
\hline \multirow{2}{*}{ Phaseolus vulgaris } & 15.09 .2015 & $\begin{array}{l}\text { Acyrthosiphon pisum } \\
\text { Aphis fabae }\end{array}$ & 480 & 38 & 7.92 \\
\hline & 20.10 .2015 & $\begin{array}{l}\text { Acyrthosiphon pisum } \\
\text { Aphis fabae }\end{array}$ & 105 & 16 & 15.24 \\
\hline \multirow[b]{2}{*}{ Cucumis sativus } & 26.08 .2015 & Aphis gossypii & 58 & 0 & 0 \\
\hline & 08.10 .2015 & $\begin{array}{l}\text { Lipaphis erysimi } \\
\text { Aphis gossypii }\end{array}$ & 342 & 31 & 9.06 \\
\hline \multirow{4}{*}{ Cucurbita pepo } & 28.07.2015 & Myzus (Nectarosiphon) persicae & 475 & 7 & 1.47 \\
\hline & 06.08 .2015 & $\begin{array}{l}\text { Myzus (Nectarosiphon) persicae } \\
\text { Aphis gossypii }\end{array}$ & 1250 & 46 & 3.68 \\
\hline & 13.08.2015 & $\begin{array}{l}\text { Aphis nasturtii } \\
\text { Myzus (Nectarosiphon) persicae }\end{array}$ & 625 & 45 & 7.20 \\
\hline & 09.09 .2015 & Aphis gossypii & 440 & 25 & 5.68 \\
\hline \multirow{3}{*}{ Cucumis melo } & 08.07 .2015 & Aphis gossypii & 1230 & 61 & 4.96 \\
\hline & 28.07 .2015 & Aphis gossypii & 185 & 16 & 8.65 \\
\hline & 19.08 .2015 & $\begin{array}{l}\text { Aphis gossypii } \\
\text { Aulacorthum solani }\end{array}$ & 306 & 23 & 7.52 \\
\hline Solanum melongena & 03.11 .2015 & Aphis gossypii & 200 & 107 & 53.50 \\
\hline
\end{tabular}

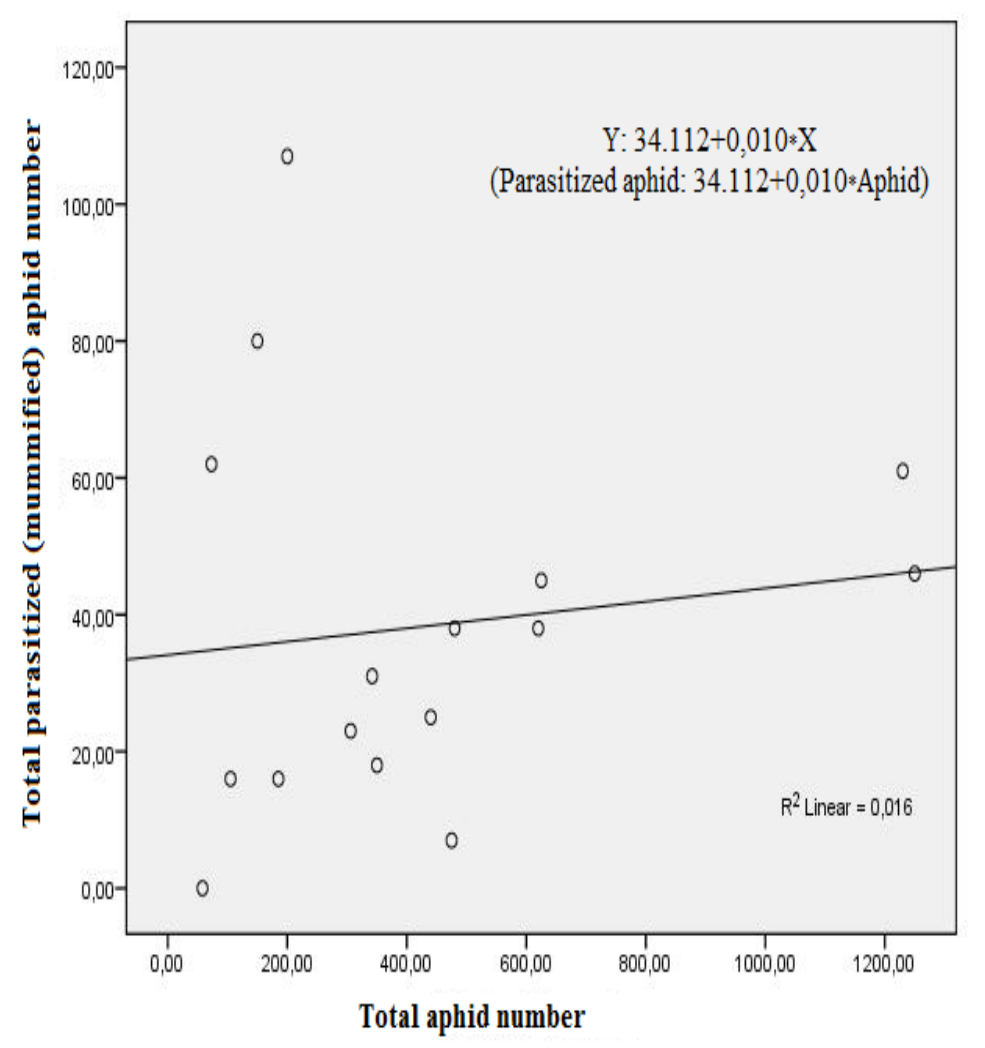

Figure 1- The relationship between the number of total parasitized (mummified) aphids and the number of total aphids in 2015

Parasitism rates in 2016 are given in Table 5. Starting from the fourth week of June and continued until the end of October, natural parasitism with aphids on vegetables was observed. Maximum parasitism was in 2016 first and fourth week October. The highest parasitism rate was recorded as $21.43 \%$ on 04 October 2016 with cultured sample, while the mean rate of parasitism was $4.39 \%$. 
Table 5- The rate of parasitism of aphids on vegetables in 2016 in Erzincan province

\begin{tabular}{|c|c|c|c|c|c|}
\hline Host plant & $\begin{array}{c}\text { Date of } \\
\text { sampling }\end{array}$ & Aphid species & $\begin{array}{c}\text { Total } \\
\text { aphid } \\
\text { number }\end{array}$ & $\begin{array}{c}\text { Total parasitized } \\
\text { (mummified) } \\
\text { aphid number }\end{array}$ & $\begin{array}{c}\text { Parasitism } \\
\text { rates }(\%)\end{array}$ \\
\hline Capsicum annuum & 28.10 .2016 & Macrosiphum euphorbiae & 45 & 5 & 11.11 \\
\hline \multirow[t]{3}{*}{ Lycopersicon esculentum } & 10.08 .2016 & Macrosiphum euphorbiae & 59 & 1 & 1.69 \\
\hline & 28.06 .2016 & Aulacorthum solani & 64 & 0 & 0 \\
\hline & 11.08 .2016 & Aphis gossypii & 2340 & 26 & 1.11 \\
\hline \multirow{7}{*}{ Cucurbita реро } & 15.08 .2016 & Aphis gossypii & 450 & 17 & 3.78 \\
\hline & 15.08 .2016 & Aphis gossypii & 650 & 12 & 1.85 \\
\hline & 23.08 .2016 & Aphis gossypii & 480 & 15 & 3.13 \\
\hline & 23.08 .2016 & Aphis gossypii & 1280 & 38 & 2.97 \\
\hline & 13.07 .2016 & Aphis gossypii & 270 & 8 & 2.96 \\
\hline & 18.08 .2016 & Aphis gossypii & 68 & 0 & 0 \\
\hline & 25.08 .2016 & Aphis gossypii & 750 & 41 & 5.47 \\
\hline \multirow{3}{*}{ Cucumis melo } & 25.08 .2016 & Aphis gossypii & 675 & 18 & 2.67 \\
\hline & 31.08 .2016 & Aphis gossypii & 30 & 1 & 3.33 \\
\hline & 04.10 .2016 & Aphis gossypii & 14 & 3 & 21.43 \\
\hline
\end{tabular}

After analyzing the 2016 data, it was found that, there was a high positive correlation $(\mathrm{r}=0.721 * *)$ between the number of total parasitized aphids and the number of total aphids. Also, there wasn't correlation between the parasitism rate and the number of total aphids. Regression analysis was performed on the data to detect the relationship between the number of total parasitized aphids and the number of total aphids and a linear relationship between them was found (Figure 2).

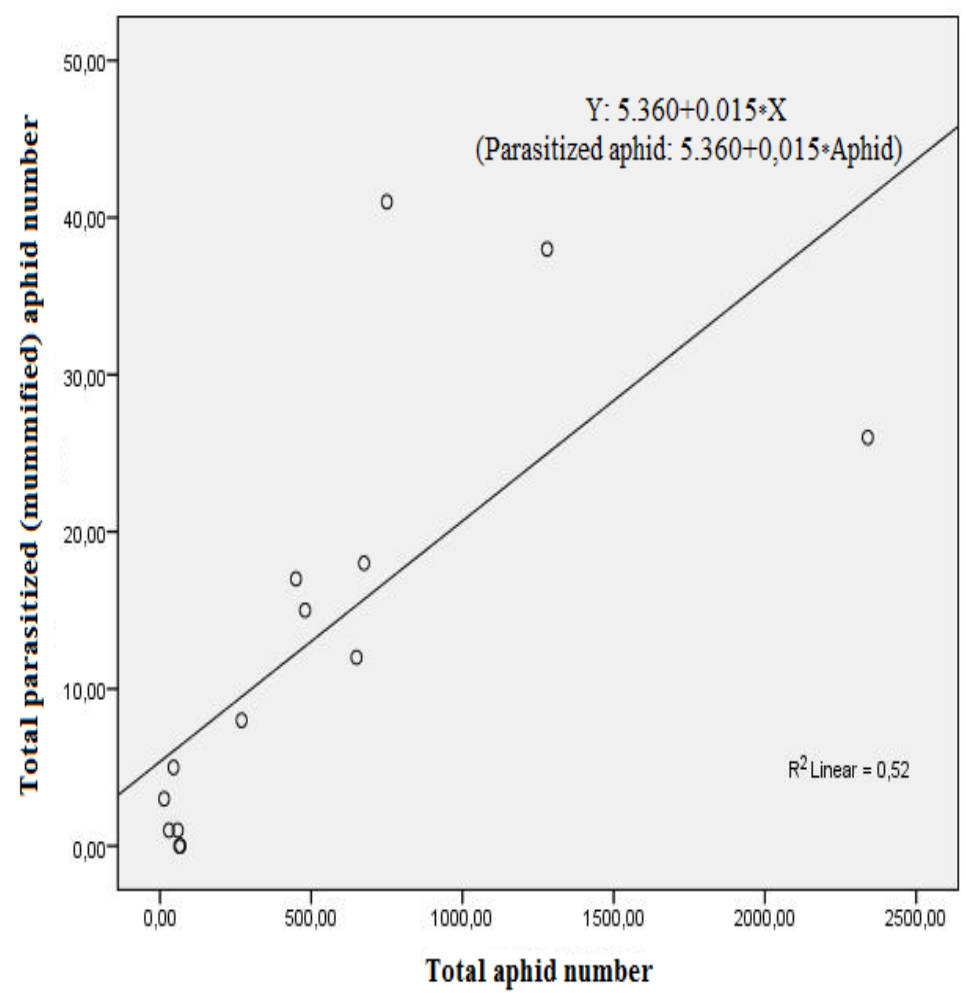

Figure 2- The relationship between the number of total parasitized (mummified) aphids and the number of total aphids in 2016

In our study, a very weak and a high positive correlation $(r=0.126-0.721)$ between total parasitized aphid number and total aphid number was found in both years. Additionally, not a correlation existed between parasitism rate and total aphid number in both consecutive years. Güçlü \& Özbek (2002), indicated a correlation between the number of parasitoid species (A. ervi parasitoids of Metopolophium dirhodum) and the number of total aphids on Rosa spp. in Erzurum province in Turkey. On the other hand, Van Veen et al. (2002), determined a very strong correlation ( $r=0.93)$ between the number of total parasitized aphids and the number of total aphids. Rakhshani et al. (2009), established that a high correlation between the number of parasitized aphids and the number of total aphids in the 5th and 6th cuts and in the 1st and 6th cuts of alfalfa respectively in 2004 ( $\mathrm{r}=0.954-$ 0.794) and $2005(r=0.933-0.794)$. Furthermore, it has been indicated a low correlation in other periods of alfalfa. Alaserhat \& Canbay (2017), conducted a study on aphid parasitoids on pepper (Capsicum annuum L.) in Erzincan province in Turkey in 2012-2013. They stated a very significant or a high positive correlation $(r=0.937-0.816)$ between the number of total parasitized 
aphids and the number of total aphids, a very weak positive correlation $(r=0.163-0.064)$ between the rates of parasitism and the number of total aphids.

\section{Conclusions}

Consequently, ten aphid, seventeen predator and seven parasitoids species were determined on vegetables. Among the aphid, predator and parasitoid species were the most common species in nature respectively A. gossypii, C. septempunctata and $P$. volucre.

Aphelinus mali (Aphelinidae: Aphelininae) was recorded as a parasitoid of L. erysimi for the first time.

Natural parasitism rates of vegetable aphids were as $17.15 \%$ in 2015 and $4.39 \%$ in 2016. In 2015, producers did not carry out the necessary processes such as fertilization, irrigation and pesticide because their selling prices were low. Even, at the end of the season they did not harvest their vegetables and left them in the field. This led to increased parasitoid and parasitism rates.

It was found that the total aphid numbers were statistically related to the total parasitized (mummified) aphid numbers, while the total aphid numbers were not statistically related to the rate of parasitism. In 2015, the relationship between the total parasitized aphid numbers and the total aphid numbers showed variability widely. Especially, since the deviations of samples taken from at the end of the season in C. annuum (03.11.2015), L. esculentum (21.10.2015) and S. melongena (03.11.2015) were high, the correlation was also low. However, in 2016, the relationship between the total parasitized aphid numbers and the total aphid numbers didn't show varriabilty, so the correlation between them was found high.

\section{Acknowledgements}

We are grateful to retired Professor Nedim UYGUN for helping to identify Coccinellidae species, Professor Ali SATAR for helping to identify Chrysopidae species, Professor Željko Tomanović for helping to identify Braconidae species, retired Professor Miktat DOĞANLAR for helping to identify Aphelinidae species, Dr. Gülten YAZICI for helping to identify Anthocoridae and Miridae species.

\section{References}

Agarwala B K \& Ghosh A K (1988). Prey records of aphidophagous Coccinellidae in India. A review and bibliography. Tropial Pest Management 34(1): 1-14

Alaserhat I (2015). The investigation on aphid (Hemiptera: Aphididae) species and their population densities, natural enemies and secondary hosts on temperate fruit species grown in Erzincan and Gümüşhane provinces. Atatürk University, Institute of Science, Plant Protection Department, Ph.D. Thesis (unpublished), Erzurum-Turkey, 320 pp

Alaserhat İ \& Canbay A (2017). Aphididae species, their parasitoids, predators, and parasitism rates on pepper (Capsicum annuum L.). Entomological News 127(1): 36-50

Anonymous (2018). Integrated pest management program. Biological Control of Aphids. http://ipm.uconn.edu/documents/raw2/htm1/734.php?aid=734 (Access date 27.02.2018)

Aslan M M (2002). Determination of Aphidoidea (Hemiptera) species and their parasitoid and predators in Kahramanmaraş province. Çukurova University, Institute of Science, Plant Protection Department, Ph.D. Thesis (unpublished), Adana-Turkey, $134 \mathrm{pp}$

Aslan M M \& Uygun N (2005). The aphidophagus coccinellid (Coleoptera: Coccinellidae) species in Kahramanmaraş, Turkey. Turkish Journal of Zoology 29: 1-8

Aslan M M \& Uygun N (2007). Afidophag syrphids (Diptera: Syrphidae) of Kahramanmaraş province of Turkey. Journal of Science and Engineering of Kahramanmaraş Sütçü İmam University 10(2): 76-81

Atwal A S, Chaudhary J P \& Ramzan M (1971). Mortality factors in the natural population of cabbage aphid, Lipaphis erysimi (Kaltenbach) (Aphididae: Hemiptera) in relations to parasites, predators and weather conditions. Indian Journal of Agricultural Science 41: 507-510

Ayyıldız Y \& Atlihan R (2006) Determination of aphid species and their natural enemies in vegetables at around Balıkesir province. Journal of Agriculture Science of Yüzüncüyıl University 16(1): 1-5

Barahoei H, Rakhshani E, Madjdzadeh S, Aliopour A, Taheri S, Nader E, Bogdanovic M, Petrovic-Obradovic O, Stary P, Kavallieratos NG \& Tomanovic Z (2013). Aphid prasitoid species (Hymenoptera: Braconidae: Aphidiinae) of central submountains of Iran. North-Western Journal of Zoology 9: 70-93

Bayram Y \& Bayhan E (2016). Life table of Aphelinus paramali Zehavi\&Rosen, 1989 (Hymenoptera: Aphelinidae), a parasite of the melon aphid, Aphis gossypii Glover, 1877 (Hemiptera, Aphididae). Acta Biologica Turcica 29(4): 111-123

Blackman R L \& Eastop V F (2018). Aphids of the world's plants. An online identification and information guide. http://www.aphidsonworldsplants.info/Introduction.htm (Access date 05.03.2018)

Brown M W \& Mathews C R (2008). Conservation biological control of spirea aphid, Aphis spiraecola (Hemiptera: Aphididae) on apple by providing natural alternative food resources. European Journal of Entomology 105: 537-540

Chaudhary H C \& Singh R (2012). Records of the predators of aphids (Hemiptera: Aphididae) in eastern Uttar Pradesh. Journal of Aphidology 25(26): 13-30

Çanakçıoglu H (1975). The Aphidoidea of Turkey. Publications of İstanbul University Faculty of Forestry, I.Ü. Publication No: 1751, F.F. Publication No: 189, Istanbul, 909 pp

Dassonville N, Thielemans T, Herbener M \& Rosemeyer V (2012). The use of mix parasitoids to control all aphid species on protected vegetable crops. Integrated Control in Protected Crops, Mediterranean Climate. IOBC-WPRS Bulletion 80: 261-266 
Düzgüneş Z \& Tuatay N (1956). Turkey aphids. Vekâlet of Agriculture, Publications of Ankara Plant Protection Institute, Volume 4, Ankara, $63 \mathrm{pp}$

Düzgüneş Z, Toros S, Kılınçer N \& Kovanc1 B (1982a). Leucopis (Diptera.: Chamaemyiidae) species which is aphid predator determined in Ankara province. Turkey Journal of Plant Protection 6: 91-96

Düzgüneş Z, Toros S, Kılınçer N \& Kovancı B (1982b). Determination of the Aphidoidea species and their parasites and predators in Ankara. Publications of General Directorate of Food and Control, Ankara-Turkey, $251 \mathrm{pp}$

Göksu M E \& Atak E D (1976). Researches on wintering status, winter hosts and population changes of peach aphids (Myzodes persicae Sulzer) and potato aphids (Macrosiphum euphorbiae Thomas) in Adapazarı Sarıkız potatoes. Plant Protection Bulletin of Turkey 16(3): $177-189$

Guo J Y, Wan F H, Dong L, Lövei G L \& Han Z J (2008). Tri-trophic interactions between Bt Cotton, the herbivore Aphis gossypii Glover (Hemiptera: Aphididae), and the predator Chrysopa pallens (Rambur) (Neuroptera: Chrysopidae). Environmental Entomology 37(1): 263270

Güçlü C \& Özbek H (2002). Effects of the parasitoids, Aphidius ervi Hal. and Praon dorsale Hal. (Hymenoptera: Aphidiidae) on Metopolophium dirhodum (Walker) (Hemiptera: Aphididae), pest of rosehip (Rosa spp.) in Erzurum. Proceedings of the Fifth Turkish National Congress of Biological Control (4-7 September 2002, Erzurum) pp. 81-88

Güleç G (2011). Determination of Aphidoidea (Hemiptera) species and natural enemies on park areas in Antalya cities. Ankara University, Institute of Science, Plant Protection Department, Ph.D. Thesis (unpublished), Ankara-Turkey $325 \mathrm{pp}$

Holman J (2009). Host plant catalog of aphids Palaearctic Region. Academy of Sciences of the Czech Republic Press, Branišovská, 1215 pp

Irshad M (2001). Aphids and their biological control of Pakistan. Pakistan Journal of Biological Sciences 45(4): 537-541

Jaferi R (2013). Feeding ability of Hippodomia variegata (Coleoptera: Coccinellidae) on Aphis fabae (Hemiptera: Aphididae). International Journal of Biology and Biological Sciences 2(1): 1-5

Kavallieratos N G, Tomanovic Z, Starý P, Athanassiou C G, Sarlis G P, Petrovic O, Niketic M \& Veroniki M A (2004). A survey of aphid parasitoids (Hymenoptera: Braconidae: Aphidiinae) of Southeastern Europe and their aphid-plant association. Applied Entomology and Zoology 39(3): 527-563

Kavallieratos N G, Tomanovic Z, Starý P, George P, Fasseas C \& Emmanouel N E (2006). A review of the genus Aphidius in Greece (Hymenoptera: Braconidae: Aphidiinae) with the description of a new species. Journal of Natural History 40(17-18): 1179-1197

Kavallieratos N G, Tomanovic Z, Stary P, Zikic V \& Petrovic-Obradovic O (2010) Parasitoids (Hymenoptera: Braconidae: Aphidiinae) attacking aphids feeding on Solanaceae and Cucurbitaceae crops in southeastern Europe: Aphidiine-Aphid-Plant Associations and Key. Annals of the Entomological Society America 103: 153-164

Lodos N (1986) Turkey Entomology II (General, Applied and Faunistic). Publications of Ege University Faculty of Agriculture, Izmir-Turkey, $580 \mathrm{pp}$

Matheus R E F (1993). Diagnosis of Plant Virus Diseases. CRS Press Inc, Boca Raton, Florida, 374 pp

Mossadegh M S, Stary P \& Salehipour H (2011). Aphid parasitoids in a dry lowland area Khuzestan, Iran (Hymenoptera, Braconidae, Aphidiinae). Asian Journal of Biological Science 4: 175-181

Nazari Y, Zamani A A, Masoumi S M, Rakhshani E, Petrovic-Obradovic O, Tomanovic S, Stary P \&Tomanovic Z (2012). Diversity and host associations of aphid parasitoids (Hymenoptera: Braconidae: Aphidiinae) in the farmland of Western Iran. Acta Entomologica Musei Nationalis Prage 52(2): 559-584

Ölmez Bayhan S (2000). Determination of Aphidoidea (Hemiptera) species and their parasitoid and predators in Diyarbakır province. Çukurova University, Institute of Science, Plant Protection Department, Master Thesis (unpublished), Adana-Turkey, $109 \mathrm{pp}$

Ölmez Bayhan S \& Ulusoy MR (2002). Determination of predators of Aphidoidea super family in Diyarbakir province, Proceedings of Fifth Turkish National Congress of Biological Control. (4-7 September 2002, Erzurum), pp. 237-246

Praslicka J \& Huszar J (2005) Hymenopteran parasitoids (Hymenoptera, Aphidiidae) on cereal aphids (Sternorrhyncha, Aphidoidea) in integrated and ecological pest management systems. Biologia-section Zoology 60(2): 227-229

Rakhshani E, Talebi A A, Kavallieratos N G, Rezwani A, Manzari S \& Tomanovic Z (2005). Parasitoid complex (Hymenoptera, Braconidae, Aphidiinae) of Aphis cracciovora Koch (Hemiptera: Aphididae) in Iran. Journal of Pest Science 78: 193-198

Rakhshani E, Talebi A A, Manzari S, Tomanovic Z, Stary P \& Rezwani A (2007). Preliminary taxonomic study of the genus Praon (Hymenoptera: Braconidae: Aphidiinae) and its host associations in Iran. Journal of Entomological Society of Iran 26(2): 19-34

Rakhshani H, Ebadi R \& Mohammadi A A (2009). Population dynamics of alfalfa aphids and their natural enemies, Isfahan, Iran. Journal of Agricultural Science Technology 11: 505-520

Rakhshani E, Stary P \& Tomanovic Z (2013). Tritrophic associations and taxonomic notes on Lysiphlebus fabarum (Marshall) (Hymenoptera: Braconidae: Aphidiinae), a keystone aphid parasitoid in Iran. Archives of Biological Science Belgrade 65(2): 667-680

Remaudière G \& Autrique A (eds) (1985). Contribution à l'écologie des aphides Africains. Étude FAO Production Végétale et Protection des Plantes 64: 1-214

Rocca M \& Messelink G J (2017). Combining lacewings and parasitoids for biological control of foxglove aphids in sweet pepper. Journal of Applied Entomology 141: 402-410

Sangün O (2010). Aphididae (Hemiptera) species harmful in lettuce cultivation areas of Eastern Mediterranean Region and researches on their controlling. Çukurova University, Institute of Science, Plant Protection Department, Master Thesis (unpublished), Adana-Turkey, 60 pp.

Sapathi C R (2009). List of predatory Coccinellidae (Coleoptera) of India and their preys: A review and bibliography. Journal of Aphidology 23(1-2): 11-42

Singh O P (1980). Reference on Lipaphis erysimi. Newsletter Aphidological Society of India 1: 7

Singh K C \& Bali G (1993). New record of Coccinellid predators on aphid (Aphis affinis and Myzus persicae) in Japanese Mint (Mentha arvensis subsp. haplocalyx var. piperascens) and Egyptian henbane (Hyoscyamus muticus). Indian Journal of Agricultural Sciences 5: 3943

Stary P \& Ghosh A K (1983). Aphid parasitoids of India and adjacent countries (Hymenoptera: Aphidiidae). Zoological Survey of India. Technical Monographs. Vol. 7: $96 \mathrm{pp}$

Stary P, Remauidiere G, Gonzales D \& Shahrokhi S (2000). A review and host associations of aphid parasitoids (Hymenoptera: Braconidae: Aphidiinae) of Iran. Parasitica 56: 15-41

Stary P (2006). Aphid parasitoids of the Czech Republic: (Hymenoptera: Braconidae, Aphidiinae). Academia Praha, 1-431 
Taheri S \& Rakhshani E (2013). Identification of aphid parasitoids (Hym., Braconidae, Aphidiinae) and determination of their relationships in southern Zagros. Journal of Plant Protection 27(1): 85-95

Talebi A A, Rakhshani E, Fathipoury Y, Stary P, Tomonovic Z \& Rajabi-Mazhar N (2009). Aphids and their parasitoids (Hym. Braconidae: Aphidiinae) associated with medicinal plants in Iran. American Eurasian Journal of Sustainable Agriculture 3: 205-219

Tepecik İ, Olcabey G, Akyıldırım H \& Görür G (2011). Aphids species which are determined in plants Karabük province and contribution to aphid fauna of turkey. Proceedings of Turkey IV. Plant Protection Congress, Kahramanmaraş. (28-30 June 2011, Maraş) pp. 201

Thompson H C \& Kelly W C (1990). Vegetable Crops (5th ed.). New Delhi: MacGraw Hill Publishing Company Ltd, 611 s

Tomanovic Z, Brajkovic M \& Krunic M (1998). A checklist of aphid parasitoids (Hymenoptera: Aphidiidae) in Yugoslavia. Acta Entomologica Serbica 3(1/2): 95-106

Tomanovic Z, Kavallieratos N G, Stary P, Athanassiou C G, Zikic V, Petrovic O \& Sarlis G P (2005). Aphidius Nees aphid parasitoids (Hymenoptera, Braconidae, Aphidiinae) in Serbia and Montenegro: Tritrophic associations and key. Acta Entomologica Serbica 8(1-2): $15-39$

Toros S, Uygun N, Ulusoy R, Satar S \& Özdemir I (2002). Aphidoidea species in the Eastern Mediterranean Region. Publications of General Directorate of Food and Control, Ankara-Turkey, $108 \mathrm{pp}$

Vandereycken A, Durieux D, Joie E, Francis F, Haubruge E \& Verheggen FJ (2015). Aphid species and associated natural enemies in field crops: What about the invasive ladybird Harmonia axyridis (Coleoptera: Coccinellidae). Entomologie Faunistique 68: 3-15

Van Veen F J F, Müller C B, Adriaanse I C T \& Godfray H C J (2002). Spatial heterogeneity in risk of secondary parasitism in a natural population of an aphid parasitoid. Journal of Animal Ecology 71: 463-469

Wiackowski S K, Wiacowska I, Werstak K \& Slusarczyk J (2001). Aphid parasitoids (Hymenoptera: Aphidiidae) of central and southern Poland. Wiadomosci Entomologiczne 20(1-2): 57-65

Yumruktepe R (1993). Researches on identification, distribution, natural enemies, population fluctuations and chemical control of harmful Aphididae (Hemiptera: Aphidiade) species in citrus orchards in Eastern Mediterranean Region. Çukurova University, Institute of Science, Plant Protection Department, Ph.D. Master Thesis (unpublished), Adana-Turkey, $127 \mathrm{pp}$

Zeren O \& Düzgüneş Z (1983). Investigations on natural enemies of Aphidoidea species which is harmful in vegetables in Çukurova Region. Turkey Journal of Plant Protection 7(3): 199-211 